\title{
The presence of intraductal carcinoma of the prostate in needle biopsy is a significant prognostic factor for prostate cancer patients with distant metastasis at initial presentation
}

\author{
Masashi Kato ${ }^{1}$, Toyonori Tsuzuki ${ }^{2}$, Kyosuke Kimura ${ }^{3}$, Akihiro Hirakawa ${ }^{4}$, \\ Fumie Kinoshita ${ }^{4}$, Naoto Sassa ${ }^{1}$, Ryo Ishida ${ }^{5}$, Akitoshi Fukatsu ${ }^{6}$, Tohru Kimura ${ }^{7}$, \\ Yasuhito Funahashi ${ }^{1}$, Yoshihisa Matsukawa ${ }^{1}$, Ryohei Hattori ${ }^{8}$ and Momokazu Gotoh ${ }^{1}$ \\ ${ }^{1}$ Department of Urology, Graduate School of Medicine, Nagoya University, Nagoya, Japan; ${ }^{2}$ Department of \\ Pathology, Japanese Red Cross Nagoya Daini Hospital, Nagoya, Japan; ${ }^{3}$ Department of Urology, National \\ Hospital Organization Nagoya Medical Center, Nagoya, Japan; ${ }^{4}$ Center for Advanced Medicine and Clinical \\ Research, Nagoya University Hospital, Nagoya, Japan; ${ }^{5}$ Department of Urology, Japanese Red Cross Nagoya \\ Daini Hospital, Nagoya, Japan; ${ }^{6}$ Department of Urology, Komaki City Hospital, Komaki, Japan; ${ }^{7}$ Department \\ of Urology, Chukyo Hospital, Nagoya, Japan and ${ }^{8}$ Department of Urology, Japanese Red Cross Nagoya Daiichi \\ Hospital, Nagoya, Japan
}

\begin{abstract}
Intraductal carcinoma of the prostate is an adverse prognostic factor in localized prostate cancer patients. However, whether it influences outcome of those patients with distant metastases discovered at initial diagnosis is unclear. Here, we evaluated whether the presence of intraductal carcinoma of the prostate in prostate needle biopsies is an adverse prognostic factor for cancer-specific survival and overall survival in such prostate cancer patients. We retrospectively enrolled 150 eligible patients. All patients received androgen-deprivation therapy and/or chemotherapy. Their age, performance status, pain, metastatic sites, clinical T stage, serum prostatespecific antigen, alkaline phosphatase, hemoglobin, Gleason score, and the presence of Gleason pattern 5 were analyzed. Primary end point was cancer-specific survival; secondary end points included prostate-specific antigen progression-free survival and overall survival. Fine and Gray's model and the Cox proportional hazards model were used as statistical tests. Intraductal carcinoma of the prostate was detected in $100(67 \%)$ patients. At a median follow-up of 38 months, 79 patients $(53 \%)$ had died of the disease and nine $(6 \%)$ had died of other causes. The average time interval to cancer-related death was 28 months. On multivariate analysis, only intraductal carcinoma of the prostate was significantly associated with cancer-specific survival $(P=0.018)$ and overall survival $(P=0.001)$, and only the presence of Gleason pattern 5 was significantly associated with prostate-specific antigen progression-free survival $(P=0.026)$. The presence of intraductal carcinoma of the prostate was the only significant prognostic parameter for cancer-specific survival and overall survival in prostate cancer patients with distant metastasis at presentation. These results may prove useful in planning future treatments.

Modern Pathology (2016) 29, 166-173; doi:10.1038/modpathol.2015.146; published online 8 January 2016
\end{abstract}

Prostate cancer is a highly heterogeneous disease, ranging from slow-growing and/or indolent tumors to rapidly progressing and fatal carcinoma. Serum

Correspondence: $\mathrm{Dr} \mathrm{T}$ Tsuzuki, $\mathrm{MD}, \mathrm{PhD}$, Department of Pathology, Japanese Red Cross Nagoya Daini Hospital, 2-9 Myoken-cho, Showa-ku, Nagoya 466-8650, Japan.

E-mail: tsuzuki@nagoya2.jrc.or.jp

Received 3 September 2015; revised 7 November 2015; accepted 8

November 2015; published online 8 January 2016 prostate-specific antigen (PSA), TNM stage, and Gleason score are well-known prognostic parameters that predict patients' clinical outcome. ${ }^{1}$ Various genomic alternations were investigated as surrogate prognostic markers in metastatic castration-resistant prostate cancer. ${ }^{2}$ However, their usefulness remains limited.

The presence of intraductal carcinoma of the prostate is a well-established adverse pathological factor. ${ }^{3-10}$ Kovi et al. first described the pathological 
features of intraductal carcinoma of the prostate as prostrate carcinoma cells dispersed within lumenspanning pre-existing prostate ducts and/or acini. ${ }^{11}$ McNeal et al. ${ }^{3}$ demonstrated that tumors with an intraductal carcinoma of the prostate component were significantly associated with advanced disease and PSA progression-free survival in radical prostatectomy cases. Recently, the presence of intraductal carcinoma of the prostate was reported to be strongly associated with high-grade and high-volume invasive prostate cancer as well as unfavorable clinical outcomes. $^{7,12}$ The latest reports demonstrated that BRCA2 mutations and PTEN loss were related to intraductal carcinoma of the prostate. ${ }^{13,14}$ Lindberg et al. ${ }^{15}$ demonstrated that prostate cancer metastasis originated from a clone derived from intraductal carcinoma of the prostate in the primary tumor.

High-grade prostatic intraepithelial neoplasia (HGPIN) is a premalignant lesion that has emerged as one of the most important differential diagnosis of intraductal carcinoma of the prostate, especially in needle biopsies. Although they are apparently morphologically similar, HGPIN usually has smaller, right-angle branches with round and smooth contours, and less cytological atypia compared with intraductal carcinoma of the prostate. ${ }^{7}$

We recently showed that the presence of intraductal carcinoma of the prostate could be an adverse prognostic parameter for both clinical progressionfree survival, cancer-specific survival, and overall survival in radical prostatectomy cases. ${ }^{6}$ Using univariate analysis, Zhao et $a .^{9}{ }^{9}$ demonstrated that intraductal carcinoma of the prostate could influence clinical outcome in prostate cancer patients with distant metastasis at initial presentation. However, whether the presence of intraductal carcinoma of the prostate is an independent prognostic parameter to predict outcome, including among clinical parameters, is still unclear. We thus aimed to evaluate whether intraductal carcinoma of the prostate detected via needle biopsy is an adverse prognostic factor for PSA progression-free survival, cancerspecific survival, and overall survival in prostate cancer patients with distant metastasis at initial diagnosis.

\section{Materials and methods}

\section{Patient Selection}

This retrospective study originally included 181 prostate cancer patients who had bone metastasis at initial diagnosis. All the patients underwent prostate biopsy with transrectal ultrasound at Nagoya University Hospital, Japanese Red Cross Nagoya Daini Hospital, Komaki City Hospital, Chukyo Hospital, or affiliated hospitals between 2002 and 2012. After excluding patients with missing data or slides, 150 patients were enrolled in this study. In addition to bone metastasis, 14 patients had visceral metastasis and 80 had lymph node metastasis. Computed tomography (CT) and/or magnetic resonance imaging (MRI) and bone scanning were performed at diagnosis. The clinical $\mathrm{T}$ stage of each tumor was re-assessed on the basis of the 2009 Union for International Cancer Control TNM classification. ${ }^{16}$ All patients initially received androgen-deprivation therapy consisting of a luteinizing hormone-releasing hormone agonist and/or anti-androgen drugs. Some patients received surgical castration. Indication of chemotherapy was decided per each patient's condition.

\section{Data Analysis}

All needle biopsy slides were reviewed by a single genitourinary pathologist (TT) according to the 2005 International Society of Urological Pathology grading system (2005 ISUP-modified Gleason score). ${ }^{17}$ Data on patient age, performance status, visceral metastasis, pain at diagnosis, clinical T stage, serum PSA, alkaline phosphatase, hemoglobin, biopsy 2005 ISUP-modified Gleason score $(<8 \quad v s \geq 8)$, the presence of Gleason pattern 5 , and the presence of intraductal carcinoma of the prostate were analyzed.

\section{Definition of Intraductal Carcinoma of the Prostate}

Intraductal carcinoma of the prostate was defined according to McNeal's criteria as described previously (Figure 1). ${ }^{3,6}$ Briefly, intraductal carcinoma of the prostate is exemplified by well-circumscribed lesions bound by intact basal cells distended by overtly malignant-appearing epithelial populations. These lumen-spanning lesions are found almost exclusively in close proximity to invasive tumors.

\section{Follow-Up}

Complete baseline and follow-up data were available for all the 150 patients. PSA was measured every 3 months following androgen-deprivation therapy. CT or MRI was performed at least every 6 months after patients were diagnosed as having castrationresistant prostate cancer. Bone scintigraphy was also performed when bone metastases were suspected. Clinical progression was defined as verification of local recurrence, distant metastasis, and/or newly diagnosed lymph node metastasis by any of the above imaging studies.

\section{Statistical Analysis}

The primary end point of this study was cancerspecific survival, which is defined as the time from diagnosis to death attributable to cancer-related complications. The secondary end points were PSA progression-free survival and overall survival. PSA progression-free survival was defined in accordance 

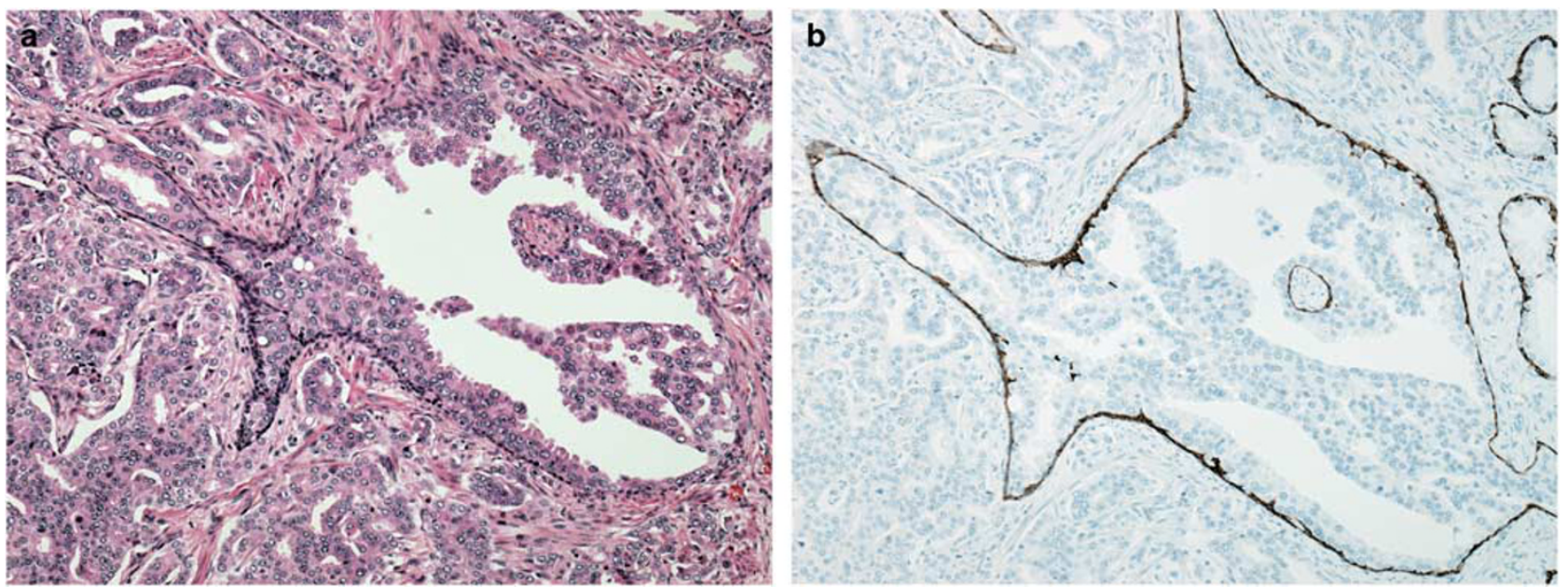

Figure 1 Representative morphological features of intraductal carcinoma of the prostate. (a) Hematoxylin and eosin staining. (b) High molecular weight cytokeratin staining. Note the presence of intact basal cells and the similar appearance of intraductal carcinoma of the prostate and invasive cancer cells.

with the Prostate Cancer Clinical Trials Working Group. ${ }^{18}$ Overall survival was defined as the time from diagnosis to death from any cause. The distribution of the clinical and pathological characteristics between intraductal carcinoma of the prostate-positive and -negative cases were compared using Fisher's exact test. Cumulative incidence curves were used in a competing-risks setting to calculate the probabilities of cancer-specific survival and PSA progression-free survival; deaths owing to other causes was treated as competing risks. ${ }^{19}$ The cumulative incidence curves for patients with or without an intraductal carcinoma of the prostate were compared using Gray's test. ${ }^{20}$ The influence of prognostic factors on cancer-specific survival was estimated by using Fine and Gray's model. ${ }^{21}$ The survival curves for the positive and negative intraductal carcinoma of the prostate cases were estimated using the Kaplan-Meier method and compared using the log-rank test. Multivariate analysis using the Cox proportional hazards model was used to evaluate the influence of prognostic factors for the overall survival. A value of $P<0.05$ was considered to be statistically significant. In the Fine and Gray's and Cox proportional hazard models, the continuous variables of PSA, hemoglobin, and alkaline phosphatase were categorized into two groups based on their median. All the statistical analyses were performed using SAS software (version 9.3; SAS Institute, Cary, NC, USA). This retrospective study was approved by the institutional review board of Nagoya University Graduate School of Medicine.

\section{Results}

Patient demographic and clinicopathological characteristics are shown in Table 1. The patients' median age was 73 years (range 50-90). The median serum PSA was $328 \mathrm{ng} / \mathrm{ml}$ (range 4.18 $10992 \mathrm{ng} / \mathrm{ml}$ ). The median-submitted number of cores was six (mean 7.3, range 1-14) and the median number of cancer-positive cores was six (mean 5.8, range 1-14). Intraductal carcinoma of the prostate was detected in $100(67 \%)$ patients, six with PSA levels $<20 \mathrm{ng} / \mathrm{ml}$ and the remaining 94 with PSA levels $>20 \mathrm{ng} / \mathrm{ml}$. Twelve patients with lower grade (clinical T2) tumors were positive for intraductal carcinoma of the prostate $(46 \%$ of all clinical T2 patients), whereas $71 \%$ of all clinical T3 and clinical T4 patients combined were positive for intraductal carcinoma of the prostate. Intraductal carcinoma of the prostate was detected in $26 \%$ of patients with 2005 ISUP-modified Gleason score $<8$ and $71 \%$ of patients with 2005 ISUP-modified Gleason score of 8 or higher. Intraductal carcinoma of the prostate was detected in $74 \%$ of patients with Gleason pattern 5 but in only $39 \%$ of patients without it. The median follow-up period was 38 months (mean 41.8 months, range 0.67-141.1 months). Patients with intraductal carcinoma of the prostate showed a statistically higher rate of anemia and higher alkaline phosphatase level $(P<0.05$, Table 1$)$.

Seventy-nine patients died of the disease and nine died of other causes. The 5- and 10-year cancerspecific survival rates in intraductal carcinoma of the prostate-positive cases were $35 \%$ and $18 \%$, and those in intraductal carcinoma of the prostatenegative cases were 69 and 53\%, respectively (Figure 2). The presence of intraductal carcinoma of the prostate was statistically correlated with cancer-specific survival $(P<0.001)$, overall survival $(P<0.001)$, and PSA progression-free survival $(P=0.001$, Figures 2 and 3). In Fine and Gray's model for cancer-specific survival, only the presence of intraductal carcinoma of the prostate was significantly associated $(P=0.018$, Table 2). In 
Table 1 Clinicopathological characteristics of the 150 patients in this study

\begin{tabular}{|c|c|c|c|c|}
\hline Variables & & $\begin{array}{l}\text { Intraductal carcinoma of the prostate } \\
\qquad(-)(\mathrm{N}=50)\end{array}$ & $\begin{array}{l}\text { Intraductal carcinoma of the prostate } \\
\qquad(+)(\mathrm{N}=100)\end{array}$ & $\mathrm{P}$-value ${ }^{\mathrm{a}}$ \\
\hline \multirow[t]{3}{*}{ Age (years) } & & & & 0.1832 \\
\hline & $\leq 65$ & $6(12 \%)$ & $22(22 \%)$ & \\
\hline & $>65$ & $44(88 \%)$ & $78(78 \%)$ & \\
\hline \multirow[t]{5}{*}{ PSA (ng/ml) } & & & & 0.0562 \\
\hline & $\leq 20$ & $7(14 \%)$ & $6(6 \%)$ & \\
\hline & $\begin{array}{l}20< \\
\leq 100\end{array}$ & $10(20 \%)$ & $24(24 \%)$ & \\
\hline & $\begin{array}{l}100< \\
\leq 1000\end{array}$ & $25(50 \%)$ & $40(40 \%)$ & \\
\hline & $1000<$ & $8(16 \%)$ & $30(30 \%)$ & \\
\hline \multirow[t]{4}{*}{ Clinical T stage } & & & & 0.3693 \\
\hline & cT2 & $14(28 \%)$ & $12(12 \%)$ & \\
\hline & сT3 & $21(42 \%)$ & $50(50 \%)$ & \\
\hline & cT4 & $15(30 \%)$ & $38(38 \%)$ & \\
\hline \multirow[t]{5}{*}{2005 ISUP-modified Gleason score } & & & & 0.001 \\
\hline & 7 & $11(22 \%)$ & $4(4 \%)$ & \\
\hline & 8 & $9(18 \%)$ & $9(9 \%)$ & \\
\hline & 9 & 27 (54\%) & $81(81 \%)$ & \\
\hline & 10 & $3(6 \%)$ & $6(6 \%)$ & \\
\hline \multirow[t]{3}{*}{ Visceral metastasis } & & & & 0.775 \\
\hline & Absent & $46(92 \%)$ & $90(90 \%)$ & \\
\hline & Present & $4(8 \%)$ & $10(10 \%)$ & \\
\hline \multirow{3}{*}{ Gleason pattern 5} & & & & 0.0005 \\
\hline & Absent & $19(38 \%)$ & $12(12 \%)$ & \\
\hline & Present & $31(62 \%)$ & $88(88 \%)$ & \\
\hline \multirow[t]{3}{*}{ Performance status } & & & & 0.4558 \\
\hline & 0 & $32(64 \%)$ & $71(71 \%)$ & \\
\hline & 1,2 & $18(36 \%)$ & $29(29 \%)$ & \\
\hline \multirow[t]{2}{*}{ Pain } & & & & 1 \\
\hline & No & $32(64 \%)$ & $64(64 \%)$ & \\
\hline \multirow{3}{*}{ Hemoglobin $(g / d l)$} & Yes & $18(36 \%)$ & $36(36 \%)$ & \\
\hline & $\geq 12.85$ & $32(64 \%)$ & 43 (43\%) & 0.0239 \\
\hline & $<12.85$ & $18(36 \%)$ & $57(57 \%)$ & \\
\hline \multirow[t]{3}{*}{ Alkaline phosphatase (IU/l) } & & & & 0.0003 \\
\hline & $\leq 398$ & $36(72 \%)$ & $40(40 \%)$ & \\
\hline & $>398$ & $14(28 \%)$ & $60(60 \%)$ & \\
\hline
\end{tabular}

${ }^{\mathrm{a}} P$-value: Fisher's exact test.

analyzing PSA progression-free survival using the same model, the presence of Gleason pattern 5 was the only significant predictive factor of PSA progression-free survival $(P=0.026)$. The presence of intraductal carcinoma of the prostate was not a significant parameter after adjustment of other variables $(P=0.188$, Table 3$)$.

The 3- and 5-year overall survival rates in patients with intraductal carcinoma of the prostate were 53 and $26 \%$, while those in patients with intraductal carcinoma of the prostate were 82 and $69 \%$, respectively. The difference of survival curves between the two groups was statistically significant $(P<0.0001$, Figure 4). Multivariate Cox regression analysis showed that the intraductal carcinoma of the prostate was the only significant parameter for predicting overall survival $(P=0.0012$, Table 4).

\section{Discussion}

The presence of intraductal carcinoma of the prostate tends to be associated with widely invasive, biologically aggressive prostate cancer. $^{7}$ Cohen et $a .^{22}$ hypothesized that intraductal carcinoma of the prostate is a late event of prostate cancer progression, during which cancer cells invade the existing duct. Therefore, detection of intraductal carcinoma of prostate in needle biopsies can be an ominous sign of prostate cancer progression. Recent reports emphasized the importance of recognizing intraductal carcinoma of the prostate in radical prostatectomy for predicting PSA progression-free survival. ${ }^{4,10,23}$ Our group was the first to report that the presence of intraductal carcinoma of the prostate is the only prognostic factor of high-risk prostate cancer on radical prostatectomy for both progression-free survival and cancer-specific survival. ${ }^{6}$ Moreover, the presence of intraductal carcinoma of the prostate is reported to be an independent prognostic parameter of early PSA progression-free survival after radiation therapy. ${ }^{5}$ Only one group reported that intraductal carcinoma of the prostate was an important adverse pathological parameter that could predict disease progression in metastatic 


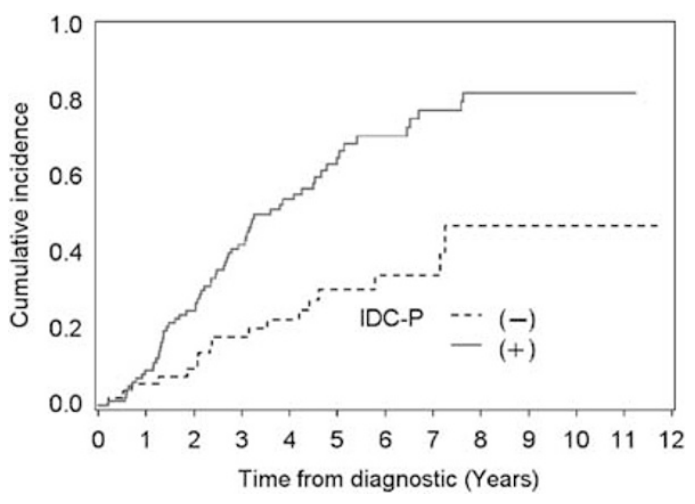

Figure 2 Cumulative incidence curves between positive and negative intraductal carcinoma of the prostate cases for cancerspecific survival. The differences were statistically significant for overall survival $(P<0.001)$.

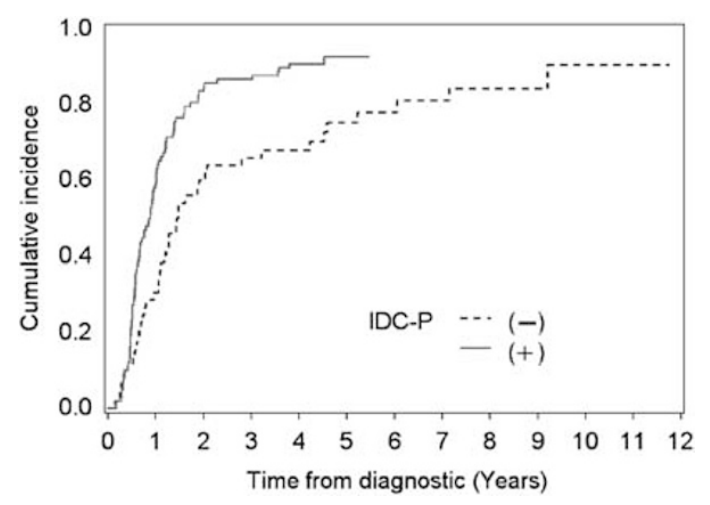

Figure 3 Cumulative incidence curves between the positive and negative intraductal carcinoma of the prostate cases for prostatespecific antigen progression-free survival. The differences were statistically significant for prostate-specific antigen progressionfree survival $(P=0.001)$.

Table 2 Cancer-specific survival and variables

\begin{tabular}{|c|c|c|c|c|}
\hline Variables & & $\mathrm{N}$ & $H R(95 \% C I)$ & $\mathrm{P}$-value \\
\hline \multirow[t]{2}{*}{ Intraductal carcinoma of the prostate } & Absent & 50 & 1 & \\
\hline & Present & 100 & $2.13(1.14-3.99)$ & 0.0181 \\
\hline \multirow[t]{2}{*}{ PSA } & $\leq 328$ & 75 & 1 & \\
\hline & $328<$ & 75 & $0.93(0.53-1.65)$ & 0.8115 \\
\hline \multirow[t]{2}{*}{ Clinical T stage } & $\mathrm{cT} 1,2,3$ & 97 & 1 & \\
\hline & cT4 & 53 & $1.20(0.74-1.92)$ & 0.4629 \\
\hline \multirow{2}{*}{2005 ISUP-modified Gleason score } & $<8$ & 15 & 1 & \\
\hline & $\geq 8$ & 135 & $0.63(0.20-2.01)$ & 0.4328 \\
\hline \multirow[t]{2}{*}{ Visceral metastasis } & Absent & 136 & 1 & \\
\hline & Present & 14 & $1.11(0.46-2.70)$ & 0.8158 \\
\hline \multirow[t]{2}{*}{ Gleason pattern 5} & Absent & 31 & 1 & \\
\hline & Present & 119 & $1.71(0.70-4.18)$ & 0.2360 \\
\hline \multirow[t]{2}{*}{ Performance status } & 0 & 103 & 1 & \\
\hline & 1,2 & 47 & $1.09(0.64-1.87)$ & 0.7538 \\
\hline \multirow[t]{2}{*}{ Pain } & No & 96 & 1 & \\
\hline & Yes & 54 & $0.94(0.55-1.60)$ & 0.8224 \\
\hline \multirow[t]{2}{*}{ Hemoglobin (g/dl) } & $\geq 12.85$ & 79 & 1 & \\
\hline & $<12.85$ & 71 & $1.61(0.95-2.74)$ & 0.0772 \\
\hline \multirow[t]{2}{*}{ Alkaline phosphatase (IU/l) } & $\leq 398$ & 63 & 1 & \\
\hline & $>398$ & 87 & $1.42(0.88-2.30)$ & 0.1567 \\
\hline
\end{tabular}

prostate cancer patients, ${ }^{9,24}$ however, their results relied on univariate analysis. We showed that the incidence of intraductal carcinoma of the prostate was $36.3 \%$ in needle biopsies and 50.5\% in radical prostatectomy specimens of high-risk prostate cancer patients, ${ }^{6}$ and that the incidence rose to $67 \%$ in patients with distant metastasis at initial diagnosis. Visceral metastasis, performance status, pain, and hemoglobin and alkaline phosphatase levels were proposed as prognostic parameters for overall survival in the TAX327 study. ${ }^{25,26}$ We demonstrated that the presence of intraductal carcinoma of the prostate on needle biopsy was the strongest prognostic parameter for cancer-specific survival and overall survival among previously reported parameters, including clinical parameters, in patients with distant metastasis at initial diagnosis.
Epstein's group has proposed separate criteria for identifying intraductal carcinoma of the prostate. ${ }^{27}$ They defined intraductal carcinoma of the prostate as malignant epithelial cells filling large acini and prostatic ducts, with preservation of basal cells forming either solid or dense cribriform patterns or loose cribriform or micropapillary patterns with marked nuclear atypia (nuclei six times the normal size or larger) or comedonecrosis. They showed that most cases that fulfilled their criteria were invasive prostate adenocarcinoma that tended to be in advanced stages, with high 2005 ISUP-modified Gleason scores and poor prognoses. ${ }^{8,27}$ The criteria focused on noninvasive needle biopsy prostate cancer specimens, most of which are supposedly from high-grade prostate cancers. These criteria are therefore stricter than McNeal's criteria; however, 
Table 3 PSA progression-free survival and variables

\begin{tabular}{|c|c|c|c|c|}
\hline Variables & & $\mathrm{N}$ & $H R$ & P-value \\
\hline \multirow[t]{2}{*}{ Intraductal carcinoma of the prostate } & Absent & 50 & 1 & \\
\hline & Present & 100 & $1.35(0.86-2.10)$ & 0.1884 \\
\hline \multirow[t]{2}{*}{ PSA } & $\leq 328$ & 75 & 1 & \\
\hline & $>328$ & 75 & $0.94(0.60-1.46)$ & 0.7756 \\
\hline \multirow[t]{2}{*}{ Clinical T stage } & cT1,2,3 & 97 & 1 & \\
\hline & $\mathrm{CT} 4$ & 53 & $1.17(0.79-1.72)$ & 0.4347 \\
\hline \multirow[t]{2}{*}{2005 ISUP-modified Gleason score } & $<8$ & 15 & 1 & \\
\hline & $\geq 8$ & 135 & $1.22(0.48-3.10)$ & 0.6800 \\
\hline \multirow{2}{*}{ Visceral metastasis } & Absent & 136 & 1 & \\
\hline & Present & 14 & $1.22(0.64-2.34)$ & 0.5427 \\
\hline \multirow{2}{*}{ Gleason pattern 5} & Absent & 31 & 1 & \\
\hline & Present & 119 & $1.85(1.08-3.19)$ & 0.0257 \\
\hline \multirow[t]{2}{*}{ Performance status } & 0 & 103 & 1 & \\
\hline & 1,2 & 47 & $0.84(0.55-1.29)$ & 0.4377 \\
\hline \multirow[t]{2}{*}{ Pain } & No & 96 & 1 & \\
\hline & Yes & 54 & $1.47(0.93-2.34)$ & 0.1033 \\
\hline \multirow[t]{2}{*}{ Hemoglobin (g/dl) } & $\geq 12.85$ & 79 & 1 & \\
\hline & $<12.85$ & 71 & $1.43(0.95-2.14)$ & 0.0879 \\
\hline \multirow[t]{2}{*}{ Alkaline phosphatase (IU/l) } & $\leq 398$ & 63 & 1 & \\
\hline & $>398$ & 87 & $1.16(0.79-1.71)$ & 0.4429 \\
\hline
\end{tabular}

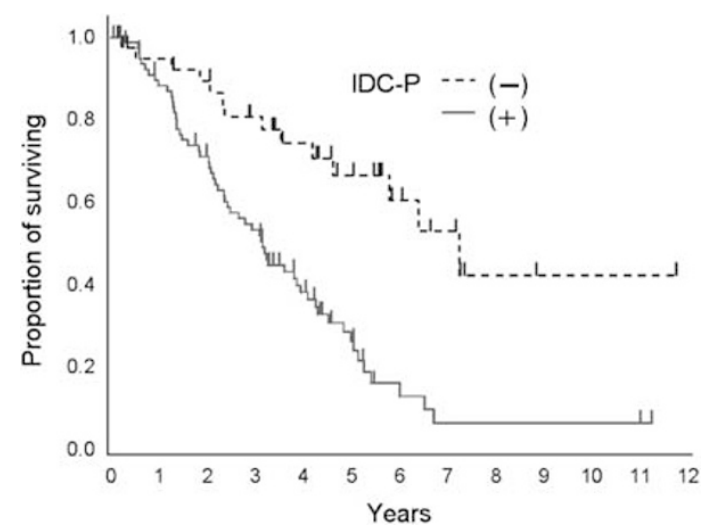

Figure 4 Survival curves of the difference between the presence and absence of intraductal carcinoma of the prostate. The differences were statistically significant for overall survival $(P<0.0001)$.

they may be less useful because many cases may be under-diagnosed. Therefore, we believe that defining intraductal carcinoma of the prostate according to McNeal's criteria is a more practical approach.

Although the presence of Gleason pattern 5 was an independent prognostic parameter for PSA progression-free survival, it was not a significant prognostic factor for cancer-specific survival and overall survival in this study. The Gleason 5 component is a well-known poor prognostic parameter for PSA progression-free survival. ${ }^{28}$ Although Chen et al. analyzed PSA progression-free survival outcomes according to 2005 ISUP-modified Gleason scores $<7$ vs 8 or above, they did not analyze the significance of the Gleason pattern 5 component. ${ }^{24}$ Our data suggest that the presence of Gleason pattern 5 can be one of the most predictive factors for PSA progression-free survival, even in patients with distant metastasis at initial diagnosis. Therefore, Gleason pattern 5 presence should be considered a poor prognostic parameter for PSA progression-free survival in current practice at every stage. On the other hand, Gleason score, including Gleason pattern 5 component, was not a significant prognostic factor for cancer-specific survival and overall survival. Tsao et al. reported that Gleason score (8 vs 9 and 10) was a prognostic factor for cancer-specific survival by univariate analysis. ${ }^{29}$ The latest reports also showed patients with 2005 ISUP-modified Gleason score of 9-10 on biopsy tended to have more aggressive cancers than those with 2005 ISUP-modified Gleason score 8 disease as determined by multivariate Cox proportional hazards analysis of PSA progression-free survival and overall survival, although the results were not statistically significant. ${ }^{28,29}$ Further studies are necessary to evaluate the significance of Gleason scores on cancer-specific survival and overall survival.

Novel therapies have become available for patients with metastatic castration-resistant prostate cancer in the last few years, and new treatment strategies have also been suggested. Some reports proposed that chemotherapy should be considered first. The CHAARTED study and the STAMPEDE trial demonstrated that upfront chemotherapy combined with androgen-deprivation therapy could improve survival in high-volume hormone-sensitive metastatic prostate cancer. ${ }^{30,31}$ van Soest et al. ${ }^{32}$ reported that docetaxel had the most pronounced survival benefit in patients with poorly differentiated tumors (Gleason score 7-10). ${ }^{33}$ Based on our results, patients with intraductal carcinoma of the prostate detected in biopsy specimens are highly likely to obtain the greatest benefit from chemotherapy as a first-line 
Table 4 Multivariate analysis of variables predicting overall survival

\begin{tabular}{|c|c|c|c|c|}
\hline \multirow{2}{*}{ Variables } & & \multirow[b]{2}{*}{$\mathrm{N}$} & \multicolumn{2}{|c|}{ Multivariate } \\
\hline & & & $H R$ & P-value ${ }^{a}$ \\
\hline \multirow{4}{*}{$\begin{array}{l}\text { Intraductal } \\
\text { carcinoma of } \\
\text { the prostate } \\
\text { PSA }\end{array}$} & Absent & 50 & & \\
\hline & Present & 100 & $2.66(1.47-4.79)$ & 0.0012 \\
\hline & $\leq 328$ & 75 & & \\
\hline & $>328$ & 75 & $0.81(0.50-1.34)$ & 0.4177 \\
\hline \multirow[t]{2}{*}{ Clinical $\mathrm{T}$ stage } & cT1,2,3 & 97 & & \\
\hline & cT4 & 53 & $1.33(0.86-2.06)$ & 0.2053 \\
\hline 2005 ISUP-modified & $<8$ & 15 & & \\
\hline \multirow{3}{*}{$\begin{array}{l}\text { Gleason score } \\
\text { visceral metastasis }\end{array}$} & $\geqq 8$ & 135 & $0.88(0.28-2.79)$ & 0.8222 \\
\hline & Absent & 136 & & \\
\hline & Present & 14 & $1.38(0.67-2.84)$ & 0.3793 \\
\hline \multirow{2}{*}{ Gleason pattern 5} & Absent & 31 & & \\
\hline & Present & 119 & $1.45(0.69-3.07)$ & 0.3275 \\
\hline \multirow{2}{*}{ Performance status } & 0 & 103 & & \\
\hline & 1,2 & 47 & $1.25(0.77-2.02)$ & 0.3753 \\
\hline \multirow[t]{2}{*}{ Pain } & No & 96 & & \\
\hline & Yes & 54 & $0.9(0.54-1.50)$ & 0.688 \\
\hline \multirow[t]{2}{*}{ Hemoglobin (g/dl) } & $\geq 12.85$ & 79 & & \\
\hline & $<12.85$ & 71 & $1.45(0.91-2.31)$ & 0.1142 \\
\hline \multirow{2}{*}{$\begin{array}{l}\text { Alkaline phosphatase } \\
\text { (IU/l) }\end{array}$} & $\leq 398$ & 63 & & \\
\hline & $>398$ & 87 & $1.57(0.97-2.54)$ & 0.066 \\
\hline
\end{tabular}

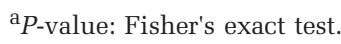

treatment instead of androgen-deprivation therapy. Prospective studies are necessary to verify this.

There are limitations in our study. First, this study was relatively small-sized and retrospective. Second, the protocols for prostate needle biopsy were not uniform across the multiple centers involved in the study. Third, androgen-deprivation therapy and/or chemotherapy were not administered uniformly because the performance status of each patient was different; in addition, the conditions of these therapies changed during the study owing to the fact that it was a long-term study. Fourth, we did not use immunohistochemical staining for confirmation of intraductal carcinoma of the prostate in needle biopsies. Although some cases might have over- or underestimated the presence of intraductal carcinoma of the prostate, most of these cases were detected only by hematoxylin and eosin-stained slides. As immunostaining for every case may not be feasible for pathologists because of their busy schedules or for economic reasons, a hematoxylin and eosin-based study is more suitable in the current practice.

Nevertheless, we believe that our study demonstrates that the presence of intraductal carcinoma of the prostate can be one of the most useful parameters to predict the outcome of patients who have distant metastasis at initial diagnosis. Our results also provide useful information for the planning of initial treatment.

\section{Conclusion}

The presence of intraductal carcinoma of the prostate in needle biopsy specimens was the only independent prognostic parameter for both cancerspecific survival and overall survival in prostate cancer patients with distant metastasis found at initial diagnosis. Physicians should be vigilant regarding follow-up of such patients and encouraged to prescribe multimodality treatments to them.

\section{Disclosure/conflict of interest}

The authors declare no conflict of interest.

\section{References}

1 Heidenreich A, Bastian PJ, Bellmunt J et al. EAU guidelines on prostate cancer. Part II: Treatment of advanced, relapsing, and castration-resistant prostate cancer. Eur Urol 2014;65:467-479.

2 Robinson D, Van Allen EM, Wu YM et al. Integrative clinical genomics of advanced prostate cancer. Cell 2015;161:1215-1228.

3 McNeal JE, Yemoto CE. Spread of adenocarcinoma within prostatic ducts and acini. Morphologic and clinical correlations. Am J Surg Pathol 1996;20: 802-814.

4 Efstathiou E, Abrahams NA, Tibbs RF et al. Morphologic characterization of preoperatively treated prostate cancer: toward a post-therapy histologic classification. Eur Urol 2010;57:1030-1038.

5 Van der Kwast T, Al Daoud N, Collette L et al. Biopsy diagnosis of intraductal carcinoma is prognostic in intermediate and high risk prostate cancer patients treated by radiotherapy. Eur J Cancer 2012;48: 1318-1325.

6 Kimura K, Tsuzuki T, Kato M et al. Prognostic value of intraductal carcinoma of the prostate in radical prostatectomy specimens. Prostate 2014;74:680-687.

7 Tsuzuki T. Intraductal carcinoma of the prostate: a comprehensive and updated review. Int J Urol 2015;22: 140-145.

8 Robinson BD, Epstein JI. Intraductal carcinoma of the prostate without invasive carcinoma on needle biopsy: emphasis on radical prostatectomy findings. J Urol 2010;184:1328-1333.

9 Zhao T, Liao B, Yao J et al. Is there any prognostic impact of intraductal carcinoma of prostate in initial diagnosed aggressively metastatic prostate cancer? Prostate 2015;75:225-232.

10 Trudel D, Downes MR, Sykes J et al. Prognostic impact of intraductal carcinoma and large cribriform carcinoma architecture after prostatectomy in a contemporary cohort. Eur J Cancer 2014;50: 1610-1616.

11 Kovi J, Jackson MA, Heshmat MY. Ductal spread in prostatic carcinoma. Cancer 1985;56:1566-1573.

12 Zhou M. Intraductal carcinoma of the prostate: the whole story. Pathology 2013;45:533-539.

13 Morais CL, Han JS, Gordetsky J et al. Utility of PTEN and ERG immunostaining for distinguishing high-grade PIN from intraductal carcinoma of the prostate on needle biopsy. Am J Surg Pathol 2015;39:169-178. 
14 Risbridger GP, Taylor RA, Clouston D et al. Patientderived xenografts reveal that intraductal carcinoma of the prostate is a prominent pathology in BRCA2 mutation carriers with prostate cancer and correlates with poor prognosis. Eur Urol 2015;67:496-503.

15 Lindberg J, Kristiansen A, Wiklund P et al. Tracking the origin of metastatic prostate cancer. Eur Urol 2015;67: 819-822.

16 Sobin LH, Gospodarowicz MK, Wittekind C. TNM Classification of Malignant Tumors, 7th (edn). WileyBlackwell: Oxford, UK, 2009.

17 Epstein JI, Allsbrook WC Jr, Amin MB et al. The 2005 International Society of Urological Pathology (ISUP) Consensus Conference on Gleason Grading of Prostatic Carcinoma. Am J Surg Pathol 2005;29: 1228-1242.

18 Scher HI, Halabi S, Tannock I et al. Design and end points of clinical trials for patients with progressive prostate cancer and castrate levels of testosterone: recommendations of the Prostate Cancer Clinical Trials Working Group. J Clin Oncol 2008;26: 1148-1159.

19 Gooley TA, Leisenring W, Crowley J et al. Estimation of failure probabilities in the presence of competing risks: new representations of old estimators. Stat Med 1999;18:695-706.

20 Gray RJ. A class of K-sample tests for comparing the cumulative incidence of a competing risk. Ann Stat 1988;16:1141-1154.

21 Fine JP, Gray RJ. A proportional hazards model for the subdistribution of a competing risk. J Am Stat Assoc 1999;94:496-509.

22 Cohen RJ, Wheeler TM, Bonkhoff $\mathrm{H}$ et al. A proposal on the identification, histologic reporting, and implications of intraductal prostatic carcinoma. Arch Pathol Lab Med 2007;131:1103-1109.

23 O'Brien BA, Cohen RJ, Wheeler TM et al. A post-radical-prostatectomy nomogram incorporating new pathological variables and interaction terms for improved prognosis. BJU Int 2011;107:389-395.
24 Chen Z, Chen N, Shen P et al. The presence and clinical implication of intraductal carcinoma of prostate in metastatic castration resistant prostate cancer. Prostate 2015;75:1247-1254.

25 Armstrong AJ, Garrett-Mayer E, Ou Yang YC et al. Prostate-specific antigen and pain surrogacy analysis in metastatic hormone-refractory prostate cancer. J Clin Oncol 2007;25:3965-3970.

26 Armstrong AJ, Garrett-Mayer ES, Yang YC et al. A contemporary prognostic nomogram for men with hormone-refractory metastatic prostate cancer: a TAX327 study analysis. Clin Cancer Res 2007;13: 6396-6403.

27 Guo CC, Epstein JI. Intraductal carcinoma of the prostate on needle biopsy: histologic features and clinical significance. Mod Pathol 2006;19:1528-1535.

28 Epstein JI, Zelefsky MJ, Sjoberg DD et al. A contemporary prostate cancer grading system: a validated alternative to the Gleason score. Eur Urol; advance online publication 9 July 2015 (e-pub ahead of print).

29 Tsao CK, Gray KP, Nakabayashi M et al. Patients with biopsy Gleason 9 and 10 prostate cancer have significantly worse outcomes compared to patients with Gleason 8 disease. J Urol 2015;194:91-97.

30 Bernard B, Sweeney CJ. Management of metastatic hormone-sensitive prostate cancer. Curr Urol Rep 2015;16:14.

31 Tombal B. Metastatic castration-resistant prostate cancer: Piling up the benefits of chemotherapy. Eur Urol 2015;68:236-237.

32 van Soest RJ, de Morree ES, Shen L et al. Initial biopsy Gleason score as a predictive marker for survival benefit in patients with castration-resistant prostate cancer treated with docetaxel: data from the TAX327 study. Eur Urol 2014;66:330-336.

33 James ND, Spears MR, Clarke NW et al. Survival with newly diagnosed metastatic prostate cancer in the "Docetaxel Era": Data from 917 patients in the control arm of the STAMPEDE Trial (MRC PR08, CRUK/ 06/019). Eur Urol 2015;67:1028-1038. 